\title{
Human Beings, Nature and Medicine: Perspectives of Internal Medicine
}

\author{
Key words: subclinical, life style, aging
}

In the process of the development of a disease, both genetic and environmental factors significantly contribute to the pathogenesis, although the involved fraction of both factors may vary among diseases. The genetic factors which are precisely imprinted in genomic DNA have been preserved for more than one billion years. In contrast, the environmental circumstances surrounding human beings have dramatically changed during the 20th century in accordance with the expeditious progress of science. The rapid change in our life style is likely to cause maladaptation to environmental circumstances, which consequently emerge as a disease. I would like to address the perspectives of Internal Medicine from the historical view of human beings whose lives have been influenced by genetic and environmental factors.

Acquisition of the tolerance to oxidant-stress originated a symbiosis with a microbe tolerant to the oxygen radicals, which turned out the mitochondria of advanced form of life. The mitochondria contain an abundance of anti-oxidant enzymes, e.g., Mn-superoxide dismutase (Mn-SOD), and ischemic injury causes its induction. The Mn-SOD protects cells and tissues even in the physiological condition as well as in the pathological condition.

In the long history of human beings, we have been attacked by various microbes, which might greatly influence the history in the Middle Age. Very recently, the discovery of Helicobacter pylori which was recognized as a cause of gastric ulcer and cancer, has necessitated the rewriting of medical textbooks. Hepatitis C virus (HCV) had been supposed to exist for a long time and was finally discovered in 1989. Interferon therapy provides a powerful strategy for fighting against HCV infection. However, careful study is now required for the appropriate use of interferon since the therapeutic efficacy is found to vary among virus genotypes. We undertook a study which revealed that even a temporal relapse could reduce the risk of occurrence of hepatoma as observed in complete remission. Infectious diseases will likely be a hamper to our life in the 21 st century.

Tissue fibrosis is a common cause of organ dysfunction in chronic inflammation including chronic hepatitis, chronic pulmonary diseases, cardiomyopathy and atherosclerosis. In renal diseases, glomerulosclerosis and tubulointerstitial fibrosis facilitate the deterioration of functional abnormalities. Activation of the cytokine network plays a crucial role in the process of the development of tissue fibrosis. Transforming growth factor $\beta$ (TGF $\beta$ ) is a key factor in the development of the tissue fibrosis; TGF $\beta$ is overexpressed in progressive kidney disease. We successfully inhibited the TGF $\beta$ gene expression in the glomeruli by means of HVJ-liposome-mediated transfection of antisense oligonucleotides into the kidney. Molecular intervention of a particular substance may open a new pathway to the therapy of fibrosis in chronic diseases.

Morbidity due to diabetes mellitus has exceeded $5 \%$ of the total population in Japan. More than 8,000 patients were introduced to dialysis therapy in 1996. Increment of non-insulindependent diabetes mellitus definitely results from the maladaptation to lifestyle; overeating, less exercise and psychological stresses which come from contemporary life. We recently reported that strict glycemic control ameliorates the insulin resistance in the liver and also the insulin secretion from the pancreatic $\beta$-cell. These findings encourage us to continue the daily clinical practice of patient education.

The remarkable progress of computer electronics has brought forth the development of new devices for body and brain imaging in the last two decades. Non-invasive image analysis such as computed tomography (CT), magnetic resonance imaging (MRI), PET provide precise and detailed information; SPECT also allows the functional analysis of the brain, and the brain imaging by $\mathrm{CT}$ and MRI depict asymptomatic subclinical abnormalities. Asymptomatic cerebral infarction has been highlighted because of its high incidence of recurrence of ischemic stroke. A randomized prospective controlled study on the role of carotid atherosclerosis in the pathogenesis of stroke (OSACA Study) demonstrated that carotid stenotic lesions of $>50 \%$ in diameter significantly contribute to the high incidence of stroke, indicating the clinical significance of subclinical abnormalities.

The life span of animals is determined as the period for growing plus the period for procreative capability. However, the progress of medical science confers our longevity which may exceed our given capacity of life. The numbers of aged people have been increasing and will occupy $25 \%$ of the total population in Japan in 2015. We have to face various diseases in such a long life of more than 80 years; thus the elderly are forced with the inevitable situation of living with incurable 
chronic diseases. We must consider the relationship among human beings, nature and medicine from the view of meaningful life in the forthcoming aging society.
Takanobu KAMADA, MD, Ph.D. President 1997, The Japanese Society of Internal Medicine, President, Osaka Rosai Hospital, Nagasone-cho 1179, Sakai 591-8025 